\title{
Generation of the Trigonometric Cubic B-Spline Collocation Solutions for the Kuramoto-Sivashinsky(KS) Equation
}

\author{
Ozlem Ersoy Hepson \\ Eskişehir Osmangazi University, Faculty of Science and Art, \\ Department of Mathematics-Computer, Eskişehir, Turkey
}

November 21, 2018

\begin{abstract}
A recent type of B-spline functions, namely trigonometric cubic B-splines, are adapted to the collocation method for the numerical solutions of the KuramotoSivashinsky equation. Having only first and second order derivatives of the trigonometric cubic B-splines at the nodes forces us to convert the Kuramoto-Sivashinsky equation to a coupled system of equations by reducing the order of the higher order terms. Crank-Nicolson method is applied for the time integration of the space discretized system resulted by trigonometric cubic B-spline approach. Some initial boundary value problems are solved to show the validity of the proposed method.
\end{abstract}

Keywords: Kuramoto-Sivashinsky Equation; Trigonometric cubic B-spline; collocation.

\section{Introduction}

The original form of the Kuramoto-Sivashinsky was constructed to describe pattern formations and dissipation of them in reaction-diffusion system[1]. In that study, the reductive perturbation method was implemented for deriving a scale-invariant part from original macroscopic motion equations. It was also shown that the Ginzburg-Landau equation can govern the dynamics near an instability point in many cases. The origin of persistent wave propagation in reaction-diffusion medium was explored by the same equation[2]. It was also used to explain the chaotic behavior in a distributed chemical reaction due to the unstable growth of a spatial inhomogeneity taking place in an oscillating medium[3]. Small model thermal diffusive instabilities in laminar flame fronts can also be represented by the same equation [4, 5. Nonlinear analysis of flame front stability assuming stiochiometric composition of the combustible mixture was also studied with constant-density 
model of a premixed flame[6]. The one-dimensional form

$$
u_{t}+u u_{x}+\alpha u_{x x}+\vartheta u_{x x x x}=0
$$

of the equation appeared in the study [7]. Hyman and Nicolaenko characterized the transition to chaos of the solutions by numerical simulations [8]. The Weiss-TaborCarnevale technique applied to the generalised Kuramoto-Sivashinsky equation to extract some particular analytical solutions [9]. In the related literature, the methods covering simplest equation, homotopy analysis and tanh and extended tanh techniques derived to determine solitary wave, or multiple soliton solutions to the Kuramoto-Sivashinsky equation[10, 11, 12, 13. Besides the analytical solutions, many numerical techniques including Chebyshev spectral collocation[14, finite difference and collocation [15], quintic B-spline [16], radial basis meshless method of lines [17], and exponential cubic B-spline method [18] have been applied to derive the numerical solutions to Kuramoto-Sivashinsky equation.

Different from the other B-splines techniques based on classical polynomial cubic, quartic and quintic B-splines[19, 20, 21] or exponential cubic B-splines [22, the trigonometric cubic B-spline functions have recently appeared. In this study, we construct a collocation method based on trigonometric cubic B-spline functions for some initial boundary value problems for the Kuramoto-Sivashinsky equation. After reducing the order of the term with the fourth order derivative to two, we discretize the resultant system by using Crank-Nicolson method in time. Performing the linearization of the nonlinear term lead us to discretize the system by trigonometric cubic B-spline functions. As a result of adapting the initial and boundary conditions, the iteration algorithm will be ready to run.

To solve the initial value (1) numerically we first replace it by a system which is first order in the time derivative

$$
\begin{array}{r}
u_{t}+u u_{x}+\alpha v+\vartheta v_{x x}=0 \\
v-u_{x x}=0
\end{array}
$$

To complete the usual classical mathematical statement of the problem, the initial and the boundary conditions are chosen as to be

$$
u(x, 0)=u_{0}
$$

and

$$
\begin{gathered}
u\left(x_{0}, t\right)=g_{0}, u\left(x_{N}, t\right)=g_{1}, \\
u_{x}\left(x_{0}, t\right)=0, u_{x}\left(x_{N}, t\right)=0, \\
u_{x x}\left(x_{0}, t\right)=0, u_{x x}\left(x_{N}, t\right)=0 .
\end{gathered}
$$

\section{Cubic Trigonometric B-spline Collocation Method}

Consider a uniform partition of the problem domain $\left[a=x_{0}, b=x_{N}\right]$ at the knots $x_{i}$, $i=0, \ldots, N$ with mesh spacing $h=(b-a) / N$. On this partition together with additional knots $x_{-2}, x_{-1}, x_{N+1}, x_{N+2}$ outside the problem domain, $T_{i}(x)$ can be defined as 
$T_{i}(x)=\frac{1}{\gamma} \begin{cases}W^{3}\left(x_{i-2}\right), & x \in\left[x_{i-2}, x_{i-1}\right] \\ W\left(x_{i-2}\right)\left(W\left(x_{i-2}\right) Y\left(x_{i}\right)+Y\left(x_{i+1}\right) W\left(x_{i-1}\right)\right)+Y\left(x_{i+2}\right) W^{2}\left(x_{i-1}\right), & x \in\left[x_{i-1}, x_{i}\right] \\ W\left(x_{i-2}\right) Y^{2}\left(x_{i+1}\right)+Y\left(x_{i+2}\right)\left(W\left(x_{i-1}\right) Y\left(x_{i+1}\right)+Y\left(x_{i+2}\right) W\left(x_{i}\right)\right), & x \in\left[x_{i}, x_{i+1}\right] \\ Y^{3}\left(x_{i+2}\right), & x \in\left[x_{i+1}, x_{i+2}\right] \\ 0, & \text { otherwise }\end{cases}$

where $W\left(x_{i}\right)=\sin \left(\frac{x-x_{i}}{2}\right), \hat{Y}\left(x_{i}\right)=\sin \left(\frac{x_{i}-x}{2}\right), \gamma=\sin \left(\frac{h}{2}\right) \sin (h) \sin \left(\frac{3 h}{2}\right)$. The twice continuously differentiable piecewise trigonometric B-spline function set $\left\{T_{i}(x)\right\}_{i=-1}^{N+1}$, forms a basis for the functions defined in the same interval [24, 25].

$T_{i}(x)$ are twice continuously differentiable piecewise trigonometric cubic B-spline on the interval $[a, b]$. The iterative formula

$$
T_{i}^{k}(x)=\frac{\sin \left(\frac{x-x_{i}}{2}\right)}{\sin \left(\frac{x_{i+k-1}-x_{i}}{2}\right)} T_{i}^{k-1}(x)+\frac{\sin \left(\frac{x_{i+k}-x}{2}\right)}{\sin \left(\frac{x_{i+k}-x_{i+1}}{2}\right)} T_{i+1}^{k-1}(x), \quad k=2,3,4, \ldots
$$

gives the cubic B-spline trigonometric functions starting with the CTB-splines of order 1 :

$$
T_{i}^{1}(x)=\left\{\begin{array}{cc}
1, & x \in\left[x_{i}, x_{i+1}\right) \\
0 & , \text { otherwise }
\end{array}\right.
$$

The graph of the trigonometric cubic B-splines over the interval [0.1] is depicted in Fig. 1.

Fig.1: Trigonometric cubic B-splines over the interval $[0,1]$ 
The nonzero functional and derivative values of trigonometric cubic B-spline functions at the grids are given in Table 1.

Table 1: Values of $T_{i}(x)$ and its principle two derivatives at the knot points

\begin{tabular}{|l|l|l|l|}
\hline & $T_{i}\left(x_{k}\right)$ & $T_{i}^{\prime}\left(x_{k}\right)$ & $T_{i}^{\prime \prime}\left(x_{k}\right)$ \\
\hline$x_{i-2}$ & 0 & 0 & 0 \\
\hline$x_{i-1}$ & $\sin ^{2}\left(\frac{h}{2}\right) \csc (h) \csc \left(\frac{3 h}{2}\right)$ & $\frac{3}{4} \csc \left(\frac{3 h}{2}\right)$ & $\frac{3(1+3 \cos (h)) \csc ^{2}\left(\frac{h}{2}\right)}{16\left[2 \cos \left(\frac{h}{2}\right)+\cos \left(\frac{3 h}{2}\right)\right]}$ \\
\hline$x_{i}$ & $\frac{2}{1+2 \cos (h)}$ & 0 & $\frac{-3 \cot ^{2}\left(\frac{3 h}{2}\right)}{2+4 \cos (h)}$ \\
\hline$x_{i+1}$ & $\sin ^{2}\left(\frac{h}{2}\right) \csc (h) \csc \left(\frac{3 h}{2}\right)$ & $-\frac{3}{4} \csc \left(\frac{3 h}{2}\right)$ & $\frac{3(1+3 \cos (h)) \csc ^{2}\left(\frac{h}{2}\right)}{16\left[2 \cos \left(\frac{h}{2}\right)+\cos \left(\frac{3 h}{2}\right)\right]}$ \\
\hline$x_{i+2}$ & 0 & 0 & 0 \\
\hline
\end{tabular}

An approximate solution $U$ and $V$ to the unknown $u$ and $v$ is written in terms of the expansion of the CTB as

$$
U(x, t)=\sum_{i=-1}^{N+1} \delta_{i} T_{i}(x), V(x, t)=\sum_{i=-1}^{N+1} \phi_{i} T_{i}(x) .
$$

where $\delta_{i}$ and $\phi_{i}$ are time dependent parameters to be determined from the collocation points $x_{i}, i=0, \ldots, N$ and the boundary and initial conditions. The nodal values $U$ and its first and second derivatives at the knots can be found from the (7) as

$$
\begin{array}{cl}
U_{i}=\alpha_{1} \delta_{i-1}+\alpha_{2} \delta_{i}+\alpha_{1} \delta_{i+1} & V_{i}=\alpha_{1} \phi_{i-1}+\alpha_{2} \phi_{i}+\alpha_{1} \phi_{i+1} \\
U_{i}^{\prime}=\beta_{1} \delta_{i-1}-\beta_{1} \delta_{i+1} & V_{i}^{\prime}=\beta_{1} \phi_{i-1}-\beta_{1} \phi_{i+1} \\
U_{i}^{\prime \prime}=\gamma_{1} \delta_{i-1}+\gamma_{2} \delta_{i}+\gamma_{1} \delta_{i+1} & V_{i}^{\prime \prime}=\gamma_{1} \phi_{i-1}+\gamma_{2} \phi_{i}+\gamma_{1} \phi_{i+1} \\
\alpha_{1}=\sin ^{2}\left(\frac{h}{2}\right) \csc (h) \csc \left(\frac{3 h}{2}\right) & \alpha_{2}=\frac{2}{1+2 \cos (h)} \quad \beta_{1}=-\frac{3}{4} \csc \left(\frac{3 h}{2}\right) \\
\gamma_{1}=\frac{3\left((1+3 \cos (h)) \csc ^{2}\left(\frac{h}{2}\right)\right)}{16\left(2 \cos \left(\frac{h}{2}\right)+\cos \left(\frac{3 h}{2}\right)\right)} \quad \gamma_{2}=-\frac{3 \cot ^{2}\left(\frac{h}{2}\right)}{2+4 \cos (h)}
\end{array}
$$

When KS equation is space-splitted as (2), The system includes the second-order derivatives so that smooth approximation can constructed with the combination of the trigonometric cubic B-splines. The time integration of the space-splitted system (2) is performed by the Crank-Nicolson method as

$$
\begin{aligned}
\frac{U^{n+1}-U^{n}}{\Delta t}+\frac{\left(U U_{x}\right)^{n+1}+\left(U U_{x}\right)^{n}}{2}+\alpha \frac{V^{n+1}+V^{n}}{2}+\vartheta \frac{V_{x x}^{n+1}+V_{x x}^{n}}{2} & =0 \\
\frac{V^{n+1}+V^{n}}{2}-\frac{U_{x x}^{n+1}+U_{x x}^{n}}{2} & =0
\end{aligned}
$$


where $U^{n+1}=U(x,(n+1) \Delta t)$ represent the solution at the $(n+1)$ th time level. Here $t^{n+1}=t^{n}+\Delta t, \Delta t$ is the time step, superscripts denote $n$th time level, $t^{n}=n \Delta t$.

One linearize terms $\left(U U_{x}\right)^{n+1}$ and $(U U x)^{n}$ in (10) as [23]

$$
\begin{aligned}
& (U U x)^{n+1}=U^{n+1} U_{x}^{n}+U^{n} U_{x}^{n+1}-U^{n} U_{x}^{n} \\
& (U U x)^{n}=U^{n} U_{x}^{n}
\end{aligned}
$$

to obtain the time-integrated linearized the KS Equation:

$$
\begin{array}{r}
\frac{2}{\Delta t} U^{n+1}-\frac{2}{\Delta t} U^{n}+U^{n+1} U_{x}^{n}+U^{n} U_{x}^{n+1}+\alpha\left(V^{n+1}+V^{n}\right)+\vartheta\left(V_{x x}^{n+1}+V_{x x}^{n}\right)=0 \\
\frac{V^{n+1}+V^{n}}{2}-\frac{U_{x x}^{n+1}+U_{x x}^{n}}{2}=0
\end{array}
$$

To proceed with space integration of the (11), an approximation of $U^{n}$ and $V^{n}$ in terms of the unknown element parameters and trigonometric cubic B-splines separately can be written as (7). Substitute Eqs (8) into (11) and collocate the resulting the equation at the knots $x_{i}, i=0, \ldots, N$ yields a linear algebraic system of equations:

$$
\begin{aligned}
& {\left[\left(\frac{2}{\Delta t}+K_{2}\right) \alpha_{1}+K_{1} \beta_{1}\right] \delta_{m-1}^{n+1}+\left(\alpha \alpha_{1}+\vartheta \gamma_{1}\right) \phi_{m-1}^{n+1}+\left[\left(\frac{2}{\Delta t}+K_{2}\right) \alpha_{2}\right] \delta_{m}^{n+1}+\left(\alpha \alpha_{2}+\vartheta \gamma_{2}\right) \phi_{m}^{n+1}} \\
& +\left[\left(\frac{2}{\Delta t}+K_{2}\right) \alpha_{1}-K_{1} \beta_{1}\right] \delta_{m+1}^{n+1}+\left(\alpha \alpha_{1}+\vartheta \gamma_{1}\right) \phi_{m+1}^{n+1} \\
& =\frac{2}{\Delta t} \alpha_{1} \delta_{m-1}^{n}-\left(\alpha \alpha_{1}+\vartheta \gamma_{1}\right) \phi_{m-1}^{n}+\frac{2}{\Delta t} \alpha_{2} \delta_{m}^{n}-\left(\alpha \alpha_{2}+\vartheta \gamma_{2}\right) \phi_{m}^{n}+\frac{2}{\Delta t} \alpha_{1} \delta_{m+1}^{n}-\left(\alpha \alpha_{1}+v \vartheta_{1}\right) \phi_{m+1}^{n} \\
& -\gamma_{1} \delta_{m-1}^{n+1}+\alpha_{1} \phi_{m-1}^{n+1}-\gamma_{2} \delta_{m}^{n+1}+\alpha_{2} \phi_{m}^{n+1}-\gamma_{1} \delta_{m+1}^{n+1}+\alpha_{1} \phi_{m+1}^{n+1} \\
& =\gamma_{1} \delta_{m-1}^{n}-\alpha_{1} \phi_{m-1}^{n}+\gamma_{2} \delta_{m}^{n}-\alpha_{2} \phi_{m}^{n}+\gamma_{1} \delta_{m+1}^{n}-\alpha_{1} \phi_{m+1}^{n}, \quad m=0 \ldots N, n=0,1 \ldots,
\end{aligned}
$$

where

$$
\begin{aligned}
& K_{1}=\alpha_{1} \delta_{i-1}+\alpha_{2} \delta_{i}+\alpha_{1} \delta_{i+1} \\
& K_{2}=\beta_{1} \delta_{i-1}-\beta_{1} \delta_{i+1} .
\end{aligned}
$$

The system (12) can be converted the following matrices system;

$$
\mathbf{A} \mathbf{x}^{n+1}=\mathbf{B} \mathbf{x}^{n}
$$

where

$$
\mathbf{A}=\left[\begin{array}{cccccccccc}
\nu_{m 1} & \nu_{m 2} & \nu_{m 3} & \nu_{m 4} & \nu_{m 5} & \nu_{m 2} & & & & \\
-\gamma_{1} & \alpha_{1} & -\gamma_{2} & \alpha_{2} & -\gamma_{1} & \alpha_{1} & & & & \\
& & \nu_{m 1} & \nu_{m 2} & \nu_{m 3} & \nu_{m 4} & \nu_{m 5} & \nu_{m 2} & & \\
& & -\gamma_{1} & \alpha_{1} & -\gamma_{2} & \alpha_{2} & -\gamma_{1} & \alpha_{1} & & \\
& & & \ddots & \ddots & \ddots & \ddots & \ddots & \ddots & \\
& & & & \nu_{m 1} & \nu_{m 2} & \nu_{m 3} & \nu_{m 4} & \nu_{m 5} & \nu_{m 2} \\
& & & & -\gamma_{1} & \alpha_{1} & -\gamma_{2} & \alpha_{2} & -\gamma_{1} & \alpha_{1}
\end{array}\right]
$$




$$
\mathbf{B}=\left[\begin{array}{cccccccccc}
\nu_{m 6} & \nu_{m 7} & \nu_{m 8} & \nu_{m 9} & \nu_{m 6} & \nu_{m 7} & & & & \\
\gamma_{1} & -\alpha_{1} & \gamma_{2} & -\alpha_{2} & \gamma_{1} & -\alpha_{1} & & & & \\
& & \nu_{m 6} & \nu_{m 7} & \nu_{m 8} & \nu_{m 9} & \nu_{m 6} & \nu_{m 7} & & \\
& & \gamma_{1} & -\alpha_{1} & \gamma_{2} & -\alpha_{2} & \gamma_{1} & -\alpha_{1} & & \\
& & & \ddots & \ddots & \ddots & \ddots & \ddots & \ddots & \\
& & & & \nu_{m 6} & \nu_{m 7} & \nu_{m 8} & \nu_{m 9} & \nu_{m 6} & \nu_{m 7} \\
& & & & \gamma_{1} & -\alpha_{1} & \gamma_{2} & -\alpha_{2} & \gamma_{1} & -\alpha_{1}
\end{array}\right]
$$

and

$$
\begin{array}{lll}
\nu_{m 1}=\left(\frac{2}{\Delta t}+K_{2}\right) \alpha_{1}+K_{1} \beta_{1} & \nu_{m 4}=\left(\alpha \alpha_{2}+\vartheta \gamma_{2}\right) & \nu_{m 7}=-\left(\alpha \alpha_{1}+\vartheta \gamma_{1}\right) \\
\nu_{m 2}=\left(\alpha \alpha_{1}+\vartheta \gamma_{1}\right) & \nu_{m 5}=\left(\frac{2}{\Delta t}+K_{2}\right) \alpha_{1}-K_{1} \beta_{1} & \nu_{m 8}=\frac{2}{\Delta t} \alpha_{2} \\
\nu_{m 3}=\left(\frac{2}{\Delta t}+K_{2}\right) \alpha_{2} & \nu_{m 6}=\frac{2}{\Delta t} \alpha_{1} & \nu_{m 9}=-\left(\alpha \alpha_{2}+\vartheta \gamma_{2}\right)
\end{array}
$$

The system (13) consist of $2 N+2$ linear equation in $2 N+6$ unknown parameters

$$
\mathbf{x}^{n+1}=\left(\delta_{-1}^{n+1}, \phi_{-1}^{n+1}, \delta_{0}^{n+1}, \phi_{0}^{n+1}, \ldots, \delta_{n+1}^{n+1}, \phi_{n+1}^{n+1},\right) .
$$

To obtain a unique solution, an additional four constraints are needed. These are obtained from the imposition of the Robin boundary conditions so that $U_{x x}(a, t)=0, V(a, t)=0$ and $U_{x x}(b, t)=0, V(b, t)=0$ gives the following equations:

$$
\begin{aligned}
& \gamma_{1} \delta_{-1}+\gamma_{2} \delta_{0}+\gamma_{1} \delta_{1}=0 \\
& \alpha_{1} \phi_{-1}+\alpha_{2} \phi_{0}+\alpha_{1} \phi_{1}=0 \\
& \gamma_{1} \delta_{N-1}+\gamma_{2} \delta_{N}+\gamma_{1} \delta_{N+1}=0 \\
& \alpha_{1} \phi_{N-1}+\alpha_{2} \phi_{N}+\alpha_{1} \phi_{N+1}=0
\end{aligned}
$$

Elimination of the parameters $\delta_{-1}, \phi_{-1}, \delta_{N+1}, \phi_{N+1}$, from the Eq.(12), using the above equations gives a solvable system of $2 N+2$ linear equations including $2 N+2$ unknown parameters. After finding the unknown parameters via the application of a variant of Thomas algorithm, approximate solutions at the knots can be obtained by placing successive three parameters in the Eq.(8).

Initial parameters $\delta_{i}^{0}, \phi_{i}^{0}, i=-1, \ldots, N+1$ are needed to start the iteration procedure (13). Thus the following requirements help to determine initial parameters:

$$
\begin{aligned}
& U_{x x}(a, 0)=0=\gamma_{1} \delta_{-1}^{0}+\gamma_{2} \delta_{0}^{0}+\gamma_{1} \delta_{1}^{0} \\
& U_{x x}\left(x_{i}, 0\right)=\gamma_{1} \delta_{i-1}^{0}+\gamma_{2} \delta_{i}^{0}+\gamma_{1} \delta_{i+1}^{0}=u_{x x}\left(x_{i}, 0\right), i=1, \ldots, N-1 \\
& U_{x x}(b, 0)=0=\gamma_{1} \delta_{N-1}^{0}+\gamma_{2} \delta_{N}^{0}+\gamma_{1} \delta_{N+1}^{0} \\
& V(a, 0)=0=\alpha_{1} \phi_{-1}^{0}+\alpha_{2} \phi_{0}^{0}+\alpha_{1} \phi_{1}^{0} \\
& V\left(x_{i}, 0\right)=\alpha_{1} \phi_{i-1}^{0}+\alpha_{2} \phi_{i}^{0}+\alpha_{1} \phi_{i+1}^{0}=v\left(x_{i}, 0\right), i=1, \ldots, N-1 \\
& V(a, 0)=\alpha_{1} \phi_{N-1}^{0}+\alpha_{2} \phi_{N}^{0}+\alpha_{1} \phi_{N+1}^{0}
\end{aligned}
$$




\section{Numerical tests}

To see versatility of the present method, three numerical examples are studied in this section. The efficiency and accuracy of the solutions will be determined by using the global relative error using formula

$$
\mathrm{GRE}=\frac{\sum_{j=1}^{N}\left|U_{j}^{n}-u_{j}^{n}\right|}{\sum_{j=1}^{N}\left|u_{j}^{n}\right|}
$$

where $U$ denotes numerical solution and $u$ denotes analytical solution.

Numerical solution of KS equation (11) is obtained for $\alpha=1$ and $\vartheta=1$ with the exact solution given by

$$
u(x, t)=b+\frac{15}{19} d\left[e \tanh \left(k\left(x-b t-x_{0}\right)\right)+f \tanh ^{3}\left(k\left(x-b t-x_{0}\right)\right)\right]
$$

the initial condition is taken from the exact solution together with boundary conditions given by (44). This example is studied in [26, 16, 27]. The above solution models the shock wave propagation with the speed $b$ and initial position $x_{0}$. We have considered domain as $\left[x_{0}, x_{N}\right]=[-30,30]$ with time step $\Delta t=0.01$ and number of partitions as 150 . In order to compare the solutions with [16] and [27] we have taken $b=5, k=\frac{1}{2} \sqrt{\frac{11}{19}}, x_{0}=-12$, $d=\sqrt{\frac{11}{19}}, e=-9, f=11$. Table 2 gives a comparison between the global relative error found by our method and by Quintic B-spline collocation method [16] and by Lattice Boltzmann method [27].

The numerical results are plotted at different time step for $\Delta t=0.005$ and $N=400$ in Fig. 2 and Fig. 3 shows projection of the solution on the x-t plane. Solution obtained by trigonometric cubic B-spline collocation method is very close to the exact solutions due to the global relative error obtained in Table 2.

Table 2: Comparison of global relative error for Example a at different time $t, N=150$

\begin{tabular}{|c|c|c|c|}
\hline Time $(t)$ & Present Method & {$[16]$} & {$[27]$} \\
\hline 1 & $2.98416 \times 10^{-5}$ & $3.81725 \times 10^{-4}$ & $6.7923 \times 10^{-4}$ \\
\hline 2 & $7.00758 \times 10^{-5}$ & $5.51142 \times 10^{-4}$ & $1.1503 \times 10^{-3}$ \\
\hline 3 & $9.51142 \times 10^{-5}$ & $7.03980 \times 10^{-4}$ & $1.5941 \times 10^{-3}$ \\
\hline 4 & $1.79237 \times 10^{-4}$ & $8.63662 \times 10^{-4}$ & $2.0075 \times 10^{-3}$ \\
\hline
\end{tabular}


Figure 2: Solutions of KS equation

Figure3: Projected solutions on $x t-$ plane

(b) This example represents chaotic behaviors with the initial condition,

$$
u(x, 0)=\cos \left(\frac{x}{2}\right) \sin \left(\frac{x}{2}\right)
$$

with the boundary condition

$$
u_{x x}(0, t)=0, u_{x x}(4 \pi, t)=0
$$

The computational domain $\left[x_{0}, x_{N}\right]=[0,4 \pi]$ is used with $N=512, \Delta t=0.001, \alpha=1$. It is shown that KS-Equation is highly sensitive for choice of the parameter $\vartheta$. In Figs. 4-7, we can observe the solution pattern exhibiting complete chaotic behaviors on the $x t$-plane, respectively. Figures illustrate that for the smaller value of $\vartheta$, chaotic behavior 
starts to evolve earlier and seen more complex instabilities.

Figure 4: Solutions on $x t-$ plane for $\vartheta=0.05$

Figure 5: Solutions on $x t-$ plane for $\vartheta$ 
Figure 6: Solutions on $x t-$ plane for $\vartheta=0.01$

Figure 7: Solutions on $x t-$ plane for $\vartheta$

(c) The KS equation (1) is obtained for $\alpha=1$ and $\vartheta=1$. This example represents the simplest nonlinear partial differential equation showing chaotic behavior when spatial domain is finite, with the Gaussian initial condition,

$$
u(x, 0)=-\exp \left(-x^{2}\right)
$$

with the boundary condition

$$
u\left(x_{0}, t\right)=0, u\left(x_{N}, t\right)=0
$$

The computational domain $\left[x_{0}, x_{N}\right]=[-30,30]$ with $N=120, \Delta t=0.001$. In Figs. 8 and 9 , we can observe the convergent numerical results by our trigonometric cubic B-Spline method of lines with complete chaotic behavior at $t=5$ and $t=20$, respectively. It is 
observed that the result shows same characteristics as in [16].

Figure 8: The Chaotic Solution of the KSE $t=5$ Figure 9: The Chaotic Solution of the KSE $t=20$

\section{References}

[1] Kuramoto, Y., \& Tsuzuki, T. (1975). On the formation of dissipative structures in reaction-diffusion systems reductive perturbation approach. Progress of Theoretical Physics, 54(3), 687-699.

[2] Kuramoto, Y., \& Tsuzuki, T. (1976). Persistent propagation of concentration waves in dissipative media far from thermal equilibrium. Progress of theoretical physics, $55(2), 356-369$.

[3] Kuramoto, Y. (1978). Diffusion-induced chaos in reaction systems. Progress of Theoretical Physics Supplement, 64, 346-367.

[4] Michelson, D. M., \& Sivashinsky, G. I. (1977). Nonlinear analysis of hydrodynamic instability in laminar flames-II. Numerical experiments. Acta Astronautica, 4(11-12), 1207-1221.

[5] Sivashinsky, G. I. (1977). Nonlinear analysis of hydrodynamic instability in laminar flames-I. Derivation of basic equations. Acta astronautica, 4(11-12), 1177-1206.

[6] Sivashinsky, G. I. (1980). On flame propagation under conditions of stoichiometry. SIAM Journal on Applied Mathematics, 39(1), 67-82.

[7] Sivashinsky, G. I., \& Michelson, D. M. (1980). On irregular wavy flow of a liquid film down a vertical plane. Progress of theoretical physics, 63(6), 2112-2114.

[8] Hyman, J. M., \& Nicolaenko, B. (1986). The Kuramoto-Sivashinsky equation: a bridge between PDE's and dynamical systems. Physica D: Nonlinear Phenomena, 18(1), 113-126. 
[9] Kudryashov, N. A. (1990). Exact solutions of the generalized Kuramoto-Sivashinsky equation. Physics Letters A, 147(5-6), 287-291.

[10] Kudryashov, N. A. (2005). Simplest equation method to look for exact solutions of nonlinear differential equations. Chaos, Solitons \& Fractals, 24(5), 1217-1231.

[11] Abbasbandy, S. (2008). Solitary wave solutions to the Kuramoto-Sivashinsky equation by means of the homotopy analysis method. Nonlinear Dynamics, 52(1-2), 35-40.

[12] Chen, H., \& Zhang, H. (2004). New multiple soliton solutions to the general BurgersFisher equation and the Kuramoto-Sivashinsky equation. Chaos, Solitons \& Fractals, 19(1), 71-76.

[13] Wazwaz, A. M. (2006). New solitary wave solutions to the Kuramoto-Sivashinsky and the Kawahara equations. Applied Mathematics and Computation, 182(2), 1642-1650.

[14] Khater, A. H., \& Temsah, R. S. (2008). Numerical solutions of the generalized Kuramoto-Sivashinsky equation by Chebyshev spectral collocation methods. Computers \& Mathematics with Applications, 56(6), 1465-1472.

[15] Lakestani, M., \& Dehghan, M. (2012). Numerical solutions of the generalized Kuramoto-Sivashinsky equation using B-spline functions. Applied Mathematical Modelling, 36(2), 605-617.

[16] Mittal, R. C., \& Arora, G. (2010). Quintic B-spline collocation method for numerical solution of the Kuramoto-Sivashinsky equation. Communications in Nonlinear Science and Numerical Simulation, 15(10), 2798-2808.

[17] Haq, S., Bibi, N., Tirmizi, S. I. A., \& Usman, M. (2010). Meshless method of lines for the numerical solution of generalized Kuramoto-Sivashinsky equation. Applied Mathematics and Computation, 217(6), 2404-2413.

[18] Ersoy, O., \& Dag, I. (2016). The Exponential Cubic B-Spline Collocation Method for the Kuramoto-Sivashinsky Equation. Filomat, 30(3), 853-861.

[19] Korkmaz, A., \& Dag, I. (2013). Cubic B-spline differential quadrature methods and stability for Burgers' equation. Engineering Computations, 30(3), 320-344.

[20] Korkmaz, A., \& Dag, I. (2013). Numerical simulations of boundary-forced RLW equation with cubic b-spline-based differential quadrature methods. Arabian Journal for Science and Engineering, 38(5), 1151-1160.

[21] Korkmaz, A., \& Dag, I. (2016). Quartic and quintic B-spline methods for advectiondiffusion equation. Applied Mathematics and Computation, 274, 208-219.

[22] Korkmaz, A., \& Akmaz, H. K. (2015). Numerical Simulations for Transport of Conservative Pollutants. Selcuk Journal of Applied Mathematics, 16(1). 
[23] S.G. Rubin, R.A. Graves, Cubic spline approximation for problems in fluid mechanics, Nasa TR R-436, Washington DC, 1975.

[24] Lyche, T., \& Winther, R. (1979). A stable recurrence relation for trigonometric Bsplines, Journal of Approximation theory, 25(3), 266-279.

[25] Walz, G. (1997). Identities for trigonometric B-splines with an application to curve design. BIT Numerical Mathematics, 37(1), 189-201

[26] Y. Xu, C. W. Shu, Local discontinuous Galerkin methods for the KuramotoSivashinsky equations and the Ito-type coupled KdV equations, Comput. Meth. Appl. Mech. Eng. 195 (2006) 3430-3447.

[27] H. Lai, C. Ma, Lattice Boltzmann method for the generalized Kuramoto-Sivashinsky equation, Physica A 388 (2009) 1405-1412. 